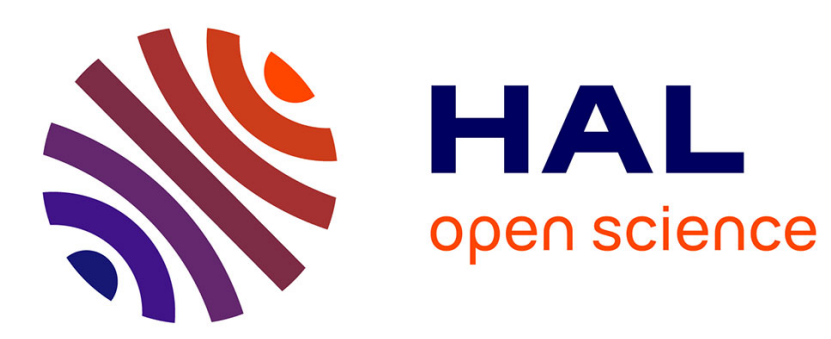

\title{
Analysing the first case of the International Criminal Court from a network-science perspective
}

Fabien Tarissan, Raphaëlle Nollez-Goldbach

\section{To cite this version:}

Fabien Tarissan, Raphaëlle Nollez-Goldbach. Analysing the first case of the International Criminal Court from a network-science perspective. Journal of Complex Networks, 2016, 4 (4), pp.616-634. 10.1093/comnet/cnw002 . hal-01367185v2

\section{HAL Id: hal-01367185 \\ https://hal.science/hal-01367185v2}

Submitted on 16 Sep 2016

HAL is a multi-disciplinary open access archive for the deposit and dissemination of scientific research documents, whether they are published or not. The documents may come from teaching and research institutions in France or abroad, or from public or private research centers.
L'archive ouverte pluridisciplinaire HAL, est destinée au dépôt et à la diffusion de documents scientifiques de niveau recherche, publiés ou non, émanant des établissements d'enseignement et de recherche français ou étrangers, des laboratoires publics ou privés. 


\title{
Analysing the first case of the International Criminal Court from a network-science perspective
}

\author{
Fabien Tarissan*1 and Raphaëlle Nollez-Goldbach ${ }^{2}$ \\ ${ }^{1}$ ISP, CNRS, Université Paris-Saclay, ENS Cachan, France \\ ${ }^{2}$ CTAD, École Normale Supérieure \& CNRS, France
}

\begin{abstract}
This paper analyses the multi-level network composed of the legal decisions taken by the International Criminal Court since its creation in 2002. As many real-world networks, legal networks lend themselves to the use of graphs in which nodes represent the decisions taken by the Court and links stand for citations between decisions. Although useful, this framework does not account for the inherent complexity and hierarchy commonly observed in real data. In the context of legal networks in particular, interactions between decisions take place at various levels, inducing a two-level structure. We propose here to rely on a hybrid version of bipartite graphs, which allows to represent different types of links in multi-level networks. We assess the relevance of this approach by analysing the hybrid structure of the first case of the Court and by confronting it with standard approaches focusing on direct citation processes. We validate the outcomes by providing juridical interpretations of the results, which shed some light on the procedural aspects of the International Criminal Court and put an emphasis on the key themes addressed by this jurisdiction. Thus, for the first time, this work converges two very different approaches to account for the multi-level complexity in legal networks.
\end{abstract}

\section{Introduction}

Many real-world networks - also referred to as complex networks - lend themselves to the use of graphs for analysing and modelling their structure. Usually, vertices of the graph stand for the nodes of the network and the edges between vertices stand for (possible) interactions between nodes of the network. This approach has proven to be useful to identify non trivial properties of the structure of networks in very different contexts, such as infrastructures of communications [44, 16], social networks [44, 29, 5], biological networks [44, 41], collaborative platforms [4, 48], co-occurrences of words [15, 39], food pairing [46, 2], etc.

Although useful, such a simple representation is not particularly close to the real structure of most of real networks. If one considers for instance actor networks [44, 28], which link actors performing in the same movies, or authoring networks [28, 29], which link authors publishing together, one would rather relate actors to the movies they performed in and authors to their papers. This observation led the community to use instead bipartite graphs, where nodes can be divided into two disjoint sets, $T$ (e.g. movies) and $\perp$ (e.g. actors), such that every link connects a node in $T$ to a node in $\perp$. Bipartite graphs are fundamental objects, which have proven to be very pertinent for both the analysis $[23,40,2]$ and modelling $[19,38]$ of complex networks as they are able to reveal patterns that could not have been detected in simple graphs.

Yet, many networks also exhibit direct interactions between nodes, which are not taken into account by bipartite graphs since links between two $\perp$ nodes are not allowed in this framework. In the example of the actor/movie networks, actors are obviously related to each other when performing in the same movies, but they might also develop social and direct relations. Although one could formally represent such a relation by the addition of an artificial $\top$ node that connects the two $\perp$ nodes, this transformation unfortunately hides important properties of the network. This calls for the use of a specific framework able to support the two types of relations. 
In the present study, we follow this approach and rely on a hybrid bipartite graph, which is composed of a traditional bipartite structure enriched with direct links between $\perp$ nodes. We address the relevance of this approach for manipulating complex networks and investigate in particular how the analysis of networks can benefit from this representation as well as how it impacts the modelling perspectives.

The International Criminal Court. To assess the interest of this hybrid approach, we compare the relevance of different frameworks for analysing the legal network composed of the decisions ruled by the International Criminal Court (ICC). Indeed, as many real-world networks, legal networks may be analysed and modelled by graphs, where nodes represent the entities that compose the networks (here the juridical decisions taken by the ICC) and links stand for relations between the entities (citations between decisions for instance). However, as stated above, this framework does not account for the inherent complexity and hierarchy of the network. In the context of legal networks in particular, interactions between decisions take place at various levels. To motivate their decisions, judges naturally rely on former decisions first level exhibiting direct interactions - but also refer to articles of the Statute of the Court second level pointing out indirect relations. This induces a two-level structure in which direct and indirect relations interplay in the ruling process. This precisely is the object which is under investigation in the present paper.

Contributions. In this paper, we analyse for the first time the legal network composed of the juridical decisions ruled by the International Criminal Court since its creation in 2002. To do so, we rely on a hybrid bipartite framework that enables us to represent both direct and indirect relations among entities of a network.

At the same time, this work provides evidence of the relevance of the hybrid bipartite approach to handle such complex networks, and sheds light on the key issues related to the proceedings of the International Criminal Court and on the interpretation of international criminal laws by the judges. In particular, we investigate which dynamics of the citation process can be highlighted and how the status of victims evolves through the decisions taken by the judges.

Note that this work builds on a previous study presented in [37] that provided preliminary results on the structure of the graph of citations and the pure bipartite structure of the ICC decisions network. The present work extends the analyses of those two structures, analyses the projections of the bipartite graph, proposes and assesses the relevance of the hybrid bipartite framework and provides juridical validations of the obtained results.

Related work. Many papers have proposed to combine law and computer science. To that regard, one of the main contributions of computer science has been to apply artificial intelligence techniques to legal networks, whether it be for highlighting legal reasoning [21, 25, 50, 49], resolving conflict [7], or extracting information from legal databases [35].

Recently, another line of research has emerged, which considers legal decisions as complex networks [33]. This originated from studying the decisions taken by the American Supreme Court $[12,17,45,13]$. In such Common Law systems, the necessity for judges to ground their decisions on precedent ones - formally referred to by the stare decicis rule [18] - has led scholars to focus exclusively on citation networks, putting aside the relation between legal decisions and articles of law. This network approach has since then been proposed in various contexts and jurisdictions $[27,1,47,10,9]$, but always focusing on citations. To that regard, it is worth citing the recent study analysing the relations between treaties, articles and legal decisions of the European Union [20] as it is an attempt to investigate the intricate and complex structure of a legal network depicting relations at different levels. However, its main contribution is to provide evidence for the "small-worl" aspect of the network and to show that the degree distribution follows a power-law.

The present paper analyses for the first time the legal network of the ICC decisions and relies on various metrics specifically defined for two-level structures. In addition, it provides explanations enabling to relate the values of the proposed metrics to the juridical interpretation 
of the decision's content. To this sense, and to the best of our knowledge, this stands out from previous studies on the subject.

In regards to the framework used in this study, the proposed hybrid bipartite structure can be related to other general frameworks proposed by social network scientists for multilevel networks, which have been applied on various contexts and have led to the proposition of dedicated models $[43,24,6,34,42]$. Taking inspiration from these approaches, we rely in this paper on a particular instance of multi-level networks as we identify only two distinct levels (sets of nodes) and allow direct links only in one of the levels. The motivation, detailed in Section 2, is driven by the structure of the information contained in the present case study.

Outline of the paper. The remainder of this paper is organised as follows. Section 2 discusses the background required throughout this paper. Section 3 presents the results of the classical approach by focusing on the graph of citations, while Section 4 presents the analysis performed on the hybrid bipartite structure and highlights its relevance. Finally, Section 5 compares the results to the ones obtained on a random network and Section 6 concludes the paper and lays some foundations for future works.

\section{A framework for the ICC decisions network}

We start by presenting the context of the study and the content of the dataset (Section 2.1) before defining the formal frameworks used in the paper to represent and analyse the data (Section 2.2).

\subsection{Legal Networks}

The contemporary evolution of international law points out the emergence of international criminal justice, whose role is to repress and redress international crimes (genocide, crimes against humanity and war crimes). Born with the Nuremberg trial after World War II, created to judge the Nazi crimes, the international criminal justice only grew recently. The first international tribunals have been created by the Security Council of the United Nations after genocide and crimes against humanity were perpetrated in ex-Yugoslavia and Rwanda during the 1990's (the International Criminal Tribunal for the former Yugoslavia in 1993 and the International Criminal Tribunal for Rwanda in 1994).

It is mostly the appearance of the International Criminal Court, that started to function in 2002, that was the landmark of the field. The ICC is indeed the first permanent international criminal court, established to help end impunity for the perpetrators of the most serious crimes of concern to the international community. 22 cases regarding 9 situations in different countries have been brought before the ICC, referred by the states themselves, the United Nations security council or the ICC prosecutor. The Court and its competences are governed by an international treaty, the Rome Statute.

The ICC's first trial started on January 2009, regarding the situation in the Democratic Republic of the Congo. Thomas Lubanga Dyilo, a former political leader and warlord, was convicted on March $14^{\text {th }} 2012$ of committing war crimes (enlisting and conscripting of children under the age of 15 years and using them to participate actively in hostilities in the context of an armed conflict). He was sentenced to 14 years of imprisonment. Both decisions were recently confirmed on appeal, on December $1^{\text {st }}$ 2014. In this paper, we only focus on the Lubanga case to concentrate on the first closed case. It is indeed the first trial in which the trial chamber of the ICC reached a verdict on the culpability of the accused ${ }^{1}$.

To rule on the Lubanga case, the ICC judges issued 2276 documents. Those decisions, main production of the ICC, are legal statements ruling on juridical issues and stand for the solutions adopted by the Court deciding on juridical issues in a case. In order to motivate their decisions, the judges may either rely on former decisions of the Court or on articles of the ICC

\footnotetext{
${ }^{1}$ Only two other cases are now terminated: the Ngujolo case (Appeal Judgement on February 27 ${ }^{\text {th }} 2015$ ) and the Katanga case (Judgement on March $7^{\text {th }} 2014$ and Sentence on May 23 2014 ).
} 
Statute they usually have to interpret. Here below is an example of such a motivation found on a footnote of decision ICC-01/04-01/06-2126-Anx (Trial chamber 1, 9 July 2009):

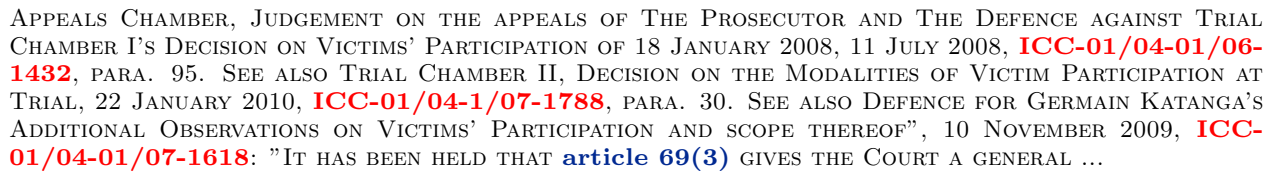

In this example, one can notice the two types of arguments used by the judges. The text clearly refers to former decisions (highlighted in red), but also refers to article of the ICC Statute (in blue).

\section{$2.2 \quad$ Frameworks}

In this section, we provide an overview of the formalisms suited to represent such a complex network. We first recall standard definitions for directed graphs (Section 2.2.1) and bipartite graphs (Section 2.2.2) before defining a hybrid version of bipartite graphs (Section 2.2.3) to overcome their limitations.

\subsubsection{Directed graphs}

As outlined in the introduction, it is quite usual to represent a network as a directed graph $G=(V, E)$, with $n=|V|$ and $m=|E|$, where a node represents a decision, identified by its ICC number, and a link between two nodes $u$ and $v$ exists if decision $u$ cites decision $v$. Note that technically, the graph is a Directed Acyclic Graph (DAG) since, for obvious reasons, the decisions can only refer to existing ones. Thus, there is no cycle in the network ${ }^{2}$.

This defines the graph of citations among decisions, which will be referred to simply as the graph of citations. This allows to compute standard metrics and compare the results to what is obtained on usual complex networks. According to standard studies, one can usually observe, for instance, that graphs are sparse, i.e. the density $\delta=\frac{2 . m}{n \cdot(n-1)}$ is very small, and their degree distribution is heterogeneous (often close to a power-law).

Another key property concerns the local density, which is meant to study how dense a neighbourhood of a node is in the graph. This concept is generally captured by the clustering coefficient $\operatorname{cc}(G)$ or the transitivity ratio $\operatorname{tr}(G)[44,32,31]$, defined formally by:

$$
\operatorname{cc}(G)=\frac{\sum_{v} \frac{\Delta(v)}{\mathrm{V}(v)}}{n} \quad \operatorname{tr}(G)=\frac{\Delta(G)}{\mathrm{V}(G)}
$$

where for each $v \in V, \Delta(v)$ denotes the number of directed triangles (sets of three nodes $u$, $v, w$, such that $(u, v),(u, w),(v, w) \in E)$ that originate at $v ; \vee(v)=\frac{d(v) \cdot(d(v)-1)}{2}$ denotes the number of pairs of neighbours of $v$, which computes the number of possible directed triangle; $\Delta(G)=\sum_{v} \Delta(v)$; and $\vee(G)=\sum_{v} \vee(v)$. Note that the clustering coefficient of a node can be defined for the in-degree and the out-degree, that is when the node is at the origin of the directed triangles (case of $u$ in the example above) and when it is at the end (case of $w$ ). Both variants make sense. They will be investigated in Section 3.3 and will be referred to as the inand out-clustering coefficients.

A classical observation in complex network studies is that all these quantities are high, at least compared to the density $\delta$ of the graph. Note however, that the meaning of the existence of such a pattern depends on the context of the network. It has been shown that it could be related to robustness properties of the network, or properties related to dynamical aspects of the networks $[26,22]$.

\subsubsection{Bipartite graphs}

As stated in the introduction, the previous formalism does not account for higher level of relations between the decisions. In particular, one does not exploit the references made to the

\footnotetext{
${ }^{2}$ All the metrics defined for directed graphs take into account this property.
} 


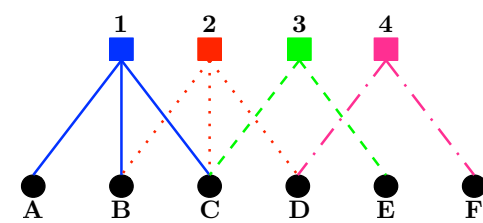

(a) bipartite graph

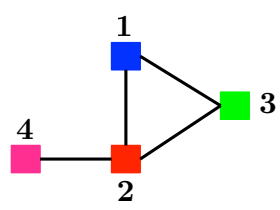

(b) T-proj.

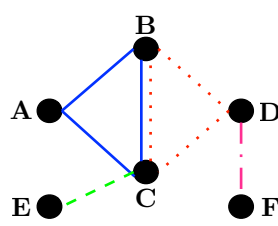

(c) $\perp$-proj.

Figure 1: Example of a bipartite graph and its $\{\top, \perp\}$-projections

articles they refer to. This two-level structure calls for a specific framework that is perfectly matched by the concept of bipartite graph.

A bipartite graph is a triplet $G_{b}=\left(\top, \perp, E_{b}\right)$, where $\top$ is the set of top nodes (here the articles of the Statute), $\perp$ the set of bottom nodes (here the decisions), and $E_{b} \subseteq T \times \perp$ the set of links that relate the decisions to the articles. The number of top and bottom nodes are respectively denoted as $n_{\top}$ and $n_{\perp}$.

Compared to standard graphs, nodes in a bipartite graph are in two disjoint sets and the links are always between a node in one set and a node in the other set. An example of a bipartite graph is given in Fig. 1(a), where $T$ nodes are depicted by squares and $\perp$ nodes by circles.

The $\perp$-projection of $G_{b}$ is the graph $G_{\perp}=\left(\perp, E_{\perp}\right)$, where two nodes (of $\perp$ ) are linked together if they have at least one neighbour in common (in $\top$ ) in $G_{v}: E_{\perp}=\{(u, v), \exists x \in$ $\top:(u, x) \in E_{b}$ and $\left.(v, x) \in E_{b}\right\}$. The T-projection is defined dually. Both projections are illustrated in Fig. 1(b) and 1(c). Thus, in our case, the $\perp$-projection corresponds to a graph of decisions, such as $G$, but a link between two decisions exists if and only if there is at least one common article to which they both refer.

Note that by projecting a bipartite graph into a simple graph, one theoretically loses information (although a recent study indicates that it is not necessarily the case when using a dual-projection approach [14]), yet such an operation allows to reuse all the metrics defined above for a standard graph. However, one can also compute specific metrics for bipartite graphs, such as the average degree of top and bottom nodes, denoted $k_{\top}$ and $k_{\perp}$ respectively, and the density of the bipartite graph, denoted $\delta_{\mathrm{b}}=\frac{m_{\mathrm{b}}}{n_{\top} \cdot n_{\perp}}$.

Those statistics are natural extensions of graph metrics. However, for the local density, there is no standard variant since by definition there is no triangle in a bipartite graph. This has led the community to propose specific metrics for the bipartite version of the clustering coefficient $[23,30]$. One can, for instance, rely on the following coefficient that tends to capture the overlapping between the neighbourhood of two nodes of $T$ :

$$
\operatorname{cc}_{\top}(u, v)=\frac{\left|N_{\top}(u) \cap N_{\top}(v)\right|}{\left|N_{\top}(u) \cup N_{\top}(v)\right|} .
$$

This coefficient is interesting as it captures the relative overlap between neighbourhoods of top nodes, i.e. $\operatorname{cc}_{\top}(u, v)$ is equal to 1 if the neighbourhood of $u$ and $v$ intersects exactly, to 0 if they do not share any neighbour. From this coefficient, it becomes natural to define the clustering coefficient related to a specific $T$ node $v$. This is given by:

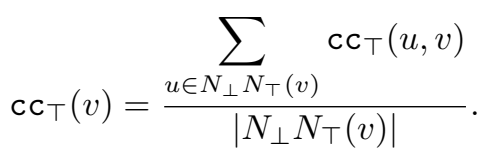

This coefficient enables us to study the distribution of this property over the top nodes as well as its correlation with the degree or other properties. Then one can naturally compute the bipartite top clustering coefficient $\mathrm{cc}_{\mathrm{bip}}$ of $G_{b}$ as the average value of $\mathrm{cc}_{\top}(v)$ over all the nodes $v$ of $T$. More formally:

$$
\mathrm{cc}_{\mathrm{bip}}\left(G_{b}\right)=\frac{1}{|\mathrm{~T}|} \sum_{v \in \mathrm{T}} \mathrm{cc} \top(v) .
$$




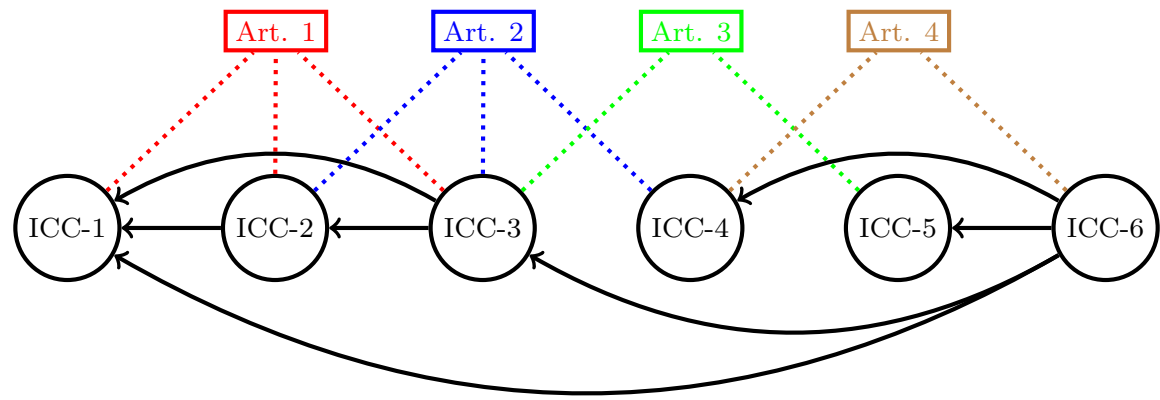

Figure 2: Example of a hybrid bipartite graph

However, it has been shown in [23] that this coefficient might miss some important properties of the overlapping between $T$ nodes in the bipartite structures. This is why the authors suggested to use the redundancy coefficient $\operatorname{rd}_{T}(v)$ of a node $v$, which focuses on the impact of removing $v$ in regards to the $\perp$-projection. Intuitively, a high value of the coefficient indicates that two $\perp$ nodes that $v$ relates are likely to be related by another $T$ node. Formally, the coefficient is given by:

$$
\operatorname{rd}_{\top}(v)=\frac{\mid\left\{\{u, w\} \in N_{\top}(v)^{2} \text { s.t. } \exists v^{\prime} \neq v,\left(v^{\prime}, u\right) \in E_{b} \text { and }\left(v^{\prime}, w\right) \in E_{b}\right\} \mid}{\frac{\left|N_{\top}(v)\right|\left(\left|N_{T}(v)\right|-1\right)}{2}} .
$$

Following this definition, we can naturally derive the redundancy coefficient $\mathrm{rd}_{\text {bip }}$ of the bipartite graph $G_{b}$, defined as the average value of the former coefficient over all $T$ nodes. More formally:

$$
\operatorname{rd}_{\mathrm{bip}}\left(G_{b}\right)=\frac{1}{|T|} \sum_{v \in T} \operatorname{rd}_{\top}(v) .
$$

\subsubsection{Hybrid bipartite graphs}

In order to overcome the limitations imposed by bipartite graphs (which does not allow to define links between $\perp$ nodes), we propose in this section a framework that enables to deal with different type of links.

A hybrid bipartite graph is formally defined by a nuplet $G_{h}=\left(\top, \perp, E_{\mathrm{b}}, E_{\mathrm{DI}}\right)$, where $\top$ is the set of top nodes (the article in our case), $\perp$ the set of bottom nodes (the decisions), $E_{\mathrm{b}} \subset T \times \perp$ is the set of links between top nodes and bottom nodes (i.e. when decisions cite articles) and $E_{\mathrm{DI}} \subset \perp \times \perp$ is the set of (directed) links between bottom nodes (i.e. when decisions cite decisions). Intuitively, top nodes indicate the presence of cliques (set of nodes all linked together) at the bottom level, the set $E_{\mathrm{b}}$ represents direct links between $\perp$ nodes and the set $E_{\mathrm{DI}}$ stands for standard bipartite links.

Compared to pure bipartite graphs, hybrid bipartite graphs thus allow for a new kind of links between $\perp$ nodes $^{3}$. Such a framework enables us to represent all the information contained in legal networks as presented in Section 2.1. An example of a hybrid bipartite graph is given in Fig 2, where $T$ nodes stand for articles of law and $\perp$ nodes for legal decisions. Links in $E_{\mathrm{DI}}$ are depicted by plain black arrows and links in $E_{\mathrm{b}}$ by dotted coloured lines.

In order to ease the reading, we will not present formally the natural extensions of the metrics defined above. However, this framework allows to focus on new properties of the structure and in particular on the interplay between the links of $E_{\mathrm{DI}}$ and the ones of $E_{\mathrm{b}}$. We can, for instance, define the notion of covering coefficient, for which given a bottom node, it computes the fraction of direct links of $E_{\mathrm{DI}}$ covered by two indirect links of $E_{\mathrm{b}}$. More formally, let $u \in \perp$ be a node

\footnotetext{
${ }^{3}$ Note that $\left(\top, \perp, E_{\mathrm{b}}\right)$ is a standard bipartite graph.
} 


\begin{tabular}{l||c|c}
\multicolumn{1}{c||}{} & \multicolumn{2}{c}{ Simple graph } \\
& Citations & Projection \\
\hline$n$ & 2276 & 2276 \\
$m$ & 8640 & 101378 \\
\hline$\delta$ & $3.4\left(10^{-3}\right)$ & $3.9\left(10^{-2}\right)$ \\
$k$ & 3.8 & 44.5 \\
$d^{+}$ & $199 / 96$ & 719 \\
\hline cc & $0.11 / 0.15$ & 0.44 \\
tr & $0.32 / 0.25$ & 0.58
\end{tabular}

\begin{tabular}{l||c} 
& Bipartite graph \\
\hline$n(\top / \perp)$ & $713 / 2276$ \\
$m_{\mathrm{b}}$ & 6670 \\
\hline$\delta_{\mathrm{b}}$ & $4.1\left(10^{-3}\right)$ \\
$k(\top / \perp)$ & $9.3 / 2.9$ \\
$d^{+}(\top / \perp)$ & $250 / 81$ \\
\hline $\mathrm{cc}_{\mathrm{bip}}$ & 0.15 \\
$r \mathrm{~d}_{\mathrm{bip}}$ & 0.69
\end{tabular}

Table 1: Global statistics for simple graphs (left) and decision/article bipartite graph (right).

of $G_{h}$, we define:

$$
\operatorname{cov}(u)=\frac{\mid\left\{v \in \perp \text { s.t. }(u, v) \in E_{\mathrm{DI}} \text { and } \exists w \in \mathrm{T},(u, w) \in E_{\mathrm{b}} \text { and }(v, w) \in E_{\mathrm{b}}\right\} \mid}{\mid\left\{v \in \perp \text { s.t. }(u, v) \in E_{\mathrm{DI}}\right\} \mid}
$$

Note that such a pattern has already proved to be of interest in former studies (see the pattern ATXAX and ATXBX of [42] for instance). In the context of the legal networks considered in this paper, this coefficient indicates the proportion for a given decision to rely on former decisions $\left(E_{\mathrm{DI}}\right)$ that deal with similar legal grounds $\left(E_{\mathrm{b}}\right)$. Taking the example of the hybrid bipartite graph of Fig. 2, one can then distinguish the case of node ICC-3, whose covering coefficient is 1 , from node ICC-6, whose covering coefficient is 0.25 .

\section{The ICC decisions network: a focus on the graph of citations}

The purpose of this section is to analyse the properties of the ICC decisions network. In particular, we question how the properties of the network are positioned in regards to the properties observed in common complex networks. To do so, we start investigating global statistics of the different structures of the network (Section 3.1) before focusing on more specific properties related to the graph of citations: the degree distribution (Section 3.2) and the local density (Section 3.3).

\subsection{Comparative analysis of the different structures}

The first statistics we focus on concern some basic properties that have been extensively investigated in real-world networks, formally presented in the previous section. Table 1 presents the results both for the simple graphs (left) and the bipartite graph (right). Regarding simple graphs, in the left table we compare the differences induced by the two kinds of relations that can link two decisions, namely the direct-citation process ${ }^{4}$ (left part) and the article-citation process (right part).

One first can see that the expected global observations made on real-world networks also stand for the graph of citations defined over the ICC decisions network. In particular, it presents a very low density $\left(3.9 \cdot 10^{-3}\right)$ with a comparatively high local density (captured by the coefficients cc and tr), which is two orders of magnitude higher. This over-representation of triangles validates the citation process as a relevant notion of a link between decisions. However, one can also notice that the maximum out-degree (as well as the maximum in-degree) observed is about two orders of magnitude higher than the average degree. This points out some heterogeneity among the citation process, which we will analyse further in the next section.

Comparing those properties to the ones computed over the graph of article-related decisions, the same conclusions can be derived, although the graph tends to be more dense than the former

\footnotetext{
${ }^{4}$ When two numbers $\mathrm{x} / \mathrm{y}$ are presented in this column, they respectively refer to the OUT links and the IN links of the graph of citations.
} 


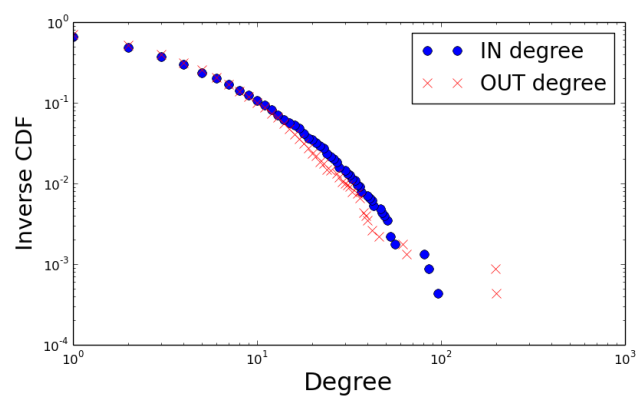

(a) Degree

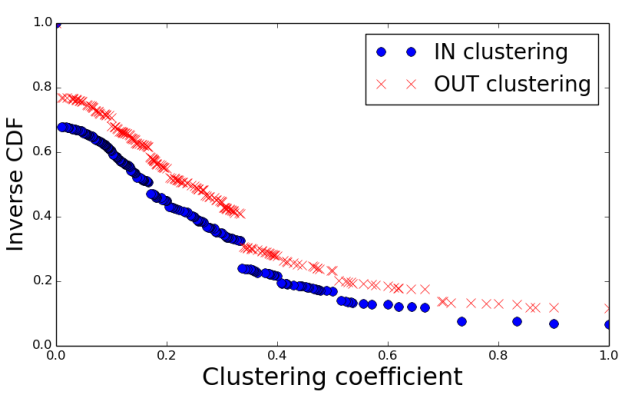

(b) Clustering coefficient

Figure 3: Inverse cumulative distributions of the degree (left) and the clustering coefficient (right) in the graph of citations.

one. In particular, the local density is very high, which is not surprising, since this graph is defined as the projection of the bipartite structure that encodes clusters of decisions.

Finally, by focusing on the global statistics of the bipartite graph, the same conclusions can be drawn. The degrees seem to be heterogeneously distributed over the structure, as suggested by the difference between the average degrees and the maximum degrees. Regarding the density, this coefficient is low compared to the local density. Yet, it is worth noticing that the two coefficients differ strongly on the value. The redundancy coefficient (0.69) is 4.6 times higher than the bipartite clustering coefficient (0.15). This raises the question of which coefficient is most relevant to discuss overlapping phenomena in bipartite structures [36]. This will be investigated more precisely in Section 4 .

In order to refine the global statistics presented above, we move now to the analysis of the value of different metrics computed over the graph of citations. Having in mind that the citation process is fundamental in elaborating a juridical decision, this section investigates which properties of the citation process the standard metrics can highlight and how it can help to focus on particular regions of the graph.

To study this question, we first present the distribution of standard metrics defined for directed graphs before deepening the investigation of the relation between degrees and clustering coefficients in the ICC decisions network.

\subsection{The degree distribution}

Figure 3(a) presents the inverse cumulative distribution of the in- and out-degree in the graph of citations. As expected, the shape of the curve (both axis are in log scale) indicates clearly a heterogeneous distribution of the degrees over several orders of magnitude. This in particular highlights nodes with high degrees in the network. Although the decisions cite and are cited 3.9 times in average (see Table 1), some decisions cite and are cited hundreds of times.

Decisions that create a precedent. From a juridical point of view, identifying the most cited decisions highlights the major substantial ICC decisions based on fundamental issues. Those decisions are called "on the merits" in contrast to the technical and procedural ones, which concern more than half of the decisions and have an in- or out-degree equal to 0 or 1 . These decisions "on the merits" set a precedent in the ICC case-law.

The most cited decision of the ICC (quoted 96 times) is the the Decision on the consequences of non-disclosure of exculpatory materials ${ }^{5}$. This decision was a major event during the Lubanga trial. The ICC judges ruled on the rupture of the conditions of a fair trial and imposed a stay on the proceedings. That decision, labelled as "urgent" in its own title, led to the decision

\footnotetext{
${ }^{5}$ Trial chamber 1, 13 June 2008, ICC-01/04-01/06-1401
} 
on the release of the accused (however, the accused continued to remain detained until the commencement of trial six months later). This was serious step back for the prosecution. The importance of that decision led the judges to comment that although they had no doubt it was necessary, "they nonetheless imposed it with great reluctance, not least because it means the Court will not make a decision on issues which are of significance to the international community, the peoples of the Democratic Republic of the Congo, the victims and the accused himself".

The second most cited decision of the ICC (quoted 86 times) is the Decision on victims participation $^{6}$. The place of victims in front of International courts is a fundamental question that judges have currently to address and one of the major stakes for the legitimacy of the ICC. Moreover, in this innovative field of international justice in general, the ICC has been the first international court alleging victims to participate to its proceedings. As the participation of the victims was included in the ICC status (art. 68 and 75), the Lubanga case-law developed it. Judges assigned to the victims, by their decisions, an active role in the proceedings. This particular decision created thus, a precedent [11]. Moreover, the fourth decision most quoted of the ICC is the appeal against that decision on victims participation, that specified it (confirming the main part, but reversing and amending others). It does point out the importance of the interpretation by the ICC judges on the matter of victims participation.

We also can mention that on the top cited decisions listed are the ones finalising the major procedural steps of trial before the ICC. For instance the Decision on the confirmation of charges $^{7}$ is the third most quoted decision of the ICC. It is the decision that formally ends the pre-trial phase. The judges confirm the existence of sufficient evidence to establish the liability of the accused, who is committed to the trial chamber for trial. Likewise, the final verdict reached by judges on the Lubanga case that convicted the accused of committing war crimes, is part of the top quoted decisions of the ICC decisions.

The judgement on culpability. Logically if we focus now on the ICC decisions that quote the most decisions, the final verdict of the Lubanga case (Judgement pursuant to Article 74 of the Statute ${ }^{8}$ ) appears widely as the main decision. Both its versions - the original in English and its translation in French, the two official languages of the Court - and the annexes the verdict comes with, listing precisely all the procedural background, are ranked as the first decisions referring to the most ICC decisions (quoting as much as 199 other decisions for the annex and 65 for the verdict). It can be easily explained since the verdict is the last decision of the trial. It then has to refer to all the previous decisions regarding the procedural steps of the trial as well as the ones ruling on the juridical fundamental issues of the case.

\subsection{Clustering coefficient}

Another strong property observed in real-networks is related to the over-representation of triangles. Figure 3(b) shows how the in-clustering and out-clustering coefficients are distributed in the network. Except for 0 (which concerns more than $20 \%$ of the nodes), this coefficient is quite uniformly distributed, although one can notice that small values seem to be more frequent. Intuitively, this shows that no strong pattern can be identified. However, this simple plot can hide different behaviour depending on the way one aggregates the data. It is also worth noticing that there is a non negligible fraction of nodes (more than 10\%) with an out-clustering of 1 , which raises the question of what kind of decisions are involved.

In order to deepen this question, Figure 4 shows the correlation between the in- and outdegree of a decision and its average in- and out-clustering coefficient. More precisely, a $(x, y)$ dot on the plot indicates that nodes having an in-degree (resp. out-degree) $x$ have an average in-clustering coefficient (resp. out-clustering) of $y$. The plot shows a clear tendency: the higher the degree, the lower the value of the coefficient. This is particularly true for small degree nodes, which have the highest clustering coefficient in average, meaning that the decisions they rely on tend to cite each-other. This is explained by the fact that small-degree decisions are

\footnotetext{
6 Trial chamber 1, 18 January 2008, ICC-01/04-01/06-1119

${ }^{7}$ Pre-trial chamber 3, 29 January 2007, ICC-01/04-01/06-803

${ }^{8}$ Trial chamber 1, 14 mars 2012, ICC-01/04-01/06-2842
} 


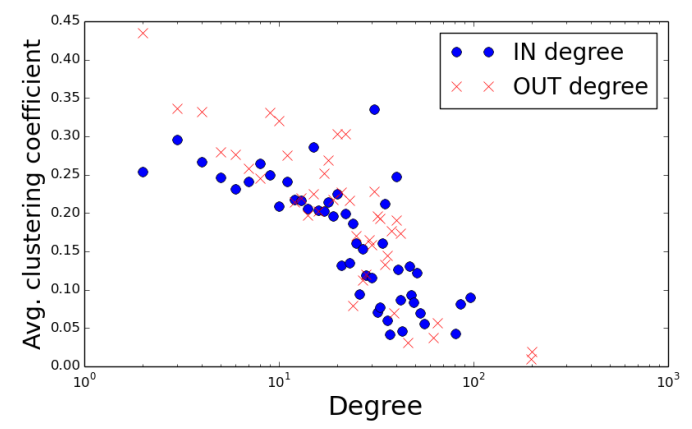

Figure 4: Correlation between degrees and clustering coefficients in the graph of citations.

by nature very specific to a part of the ICC judicial procedure andusually raise specific and technical questions, while large-degree nodes deal with different juridical issues and thus, refer to a broader scope of procedural and substantial matters.

A juridical perspective. To confirm this last statement, we first looked at the most frequent decisions (in the top ten) involved in triangles. It turns out that all of them indeed deal with substantial matters. Here again, both major decisions are the Decision on the consequences of non-disclosure of exculpatory materials and the Decision on victims participation, for which the juridical relevance has been explained previously.

The importance of the first decision and the juridical issues it ruled on is emphasised by the fact that the other major decisions related to the topic, namely the Decision on the release of Thomas Lubanga Dyilo ${ }^{9}$, that results directly from the former decision, is also part of the most frequent in the triangles. This is also the case for more procedural decisions regarding the release $^{10,11,12}$. Those decisions belong to the same procedural chain, the importance of which is fundamental from a procedural point of view (the question of whether the accused was about to be released has deeply affected the Lubanga trial) as well as from a substantial point of view (the definition of the conditions of a fair trial). This explains the presence of such decisions "on the merits" in triangles. In addition, it is worth noticing that the verdict convicting Lubanga of committing war crimes is naturally one of the most frequent decision involved in a triangle.

In regards to the counterpart of our former claim (low degree nodes with a high clustering deal with procedural aspects), it is more difficult to assess its relevance since low degree nodes are numerous (see Fig. 3(a)). Thus, we selected two extreme cases: we identified the decision with the highest in-degree (resp. out-degree) among the nodes having highest in-clustering (resp. out-clustering) coefficient. This resulted respectively in Decision on the Prosecution's Application for Leave to Appeal the "Decision on the Prosecution's Application to Lift the Stay of the Proceedings"13 and Request for Review of Detention ${ }^{14}$. The first one is a procedural decision on an application from the Prosecutor for leave to appeal against a precedent decision, the latter, regarding itself a precedent application. This structure thus, reveals the existence of a procedural chain. The second is also a procedural decision regarding the conduct of proceedings between the Pre-trial and Trial chambers. Thus, both decisions confirm the expected procedural aspect of low-degree nodes with high clusterings.

\footnotetext{
${ }^{9}$ Trial chamber 1, 2 July 2008, ICC-01/04-01/06-1418

${ }^{10}$ Trial chamber 1,2 July 2008, ICC-01/04-01/06-1417

${ }^{11}$ Appeals chamber, 14 July 2008, ICC-01/04-01/06-1434

${ }^{12}$ Trial chamber 1, 15 September 2008, ICC-01/04-01/06-1470

${ }^{13}$ Trial chamber 1, 24 September 2008, ICC-01/04-01/06-1473

${ }^{14}$ Trial chamber 1, 6 June 2007, ICC-01/04-01/06-921
} 


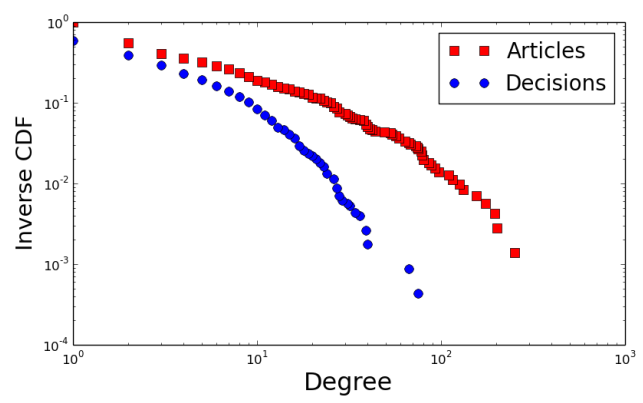

(a) Degrees

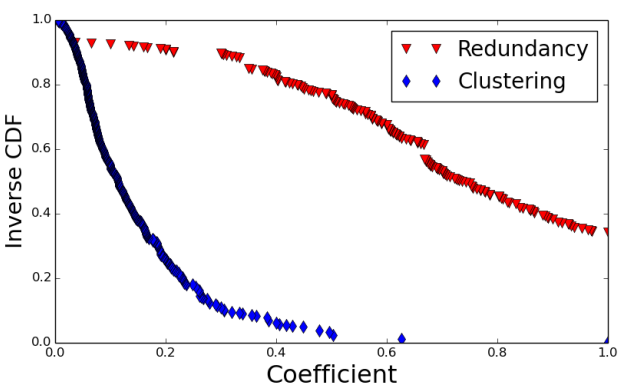

(b) Clustering and redundancy

Figure 5: Inverse cumulative distributions of degrees, clustering coefficient and redundancy coefficient in the bipartite graph.

\section{The impact of the hybrid bipartite structure}

We turn now to the analysis of the hybrid bipartite structure revealed by the ICC decisions network. The interest of studying the network from this point of view is that it allows to focus on another process used by the judges to elaborate on the ruling procedures. It highlights the role of the articles of the Statute of the Court to support the decisions and how both decisions and articles interplay. Formally, this is done by studying at the same time metrics on the bipartite graph, on its projections (Section 4.1) and on the relation between the different types of links (Section 4.2). This is what this section is devoted to.

\subsection{Interplay between the nodes}

We start by studying the distribution of different metrics defined for bipartite graphs before showing some non trivial relations between this structure and the graphs derived by its projections.

Degree distribution. We present in Figure 5(a) the inverse cumulative distribution of the degrees of both the articles and decisions in the bipartite graph. Similar to the degree distribution in the graph of citation (see Figure 3(a)), the shape of the plots (in log-log scale) indicates that the degrees are heterogeneously distributed in the bipartite network. Again, this puts an emphasis on high-degree articles and decisions that intuitively tend to address juridical issues shared by many different decisions in the Lubanga case-law. Note that the degree of a given decision in the bipartite structure has a priori no relation with its degree in the graph of citations, since citing former decisions has no relation with relying on articles of the Statute.

As expected, the ICC decisions that cite the most articles of the Statute is the verdict reached by the judges convicting the accused (both its versions in French and English). It relies on 81 articles of the Statute. Likewise, the Decision on the confirmation of charges, by which judges confirm the existence of sufficient evidence and commit the accused for trial, quotes 63 articles. Those decisions based on the highest number of articles of the Statute are both decisions that end one phase of the Lubanga case, the pre-trial and the trial. Juridical logic implies then that they respect the principle of legality and the applicable law. The motivations of the decisions have to be based on the articles of Statute, that the judges may interpret.

A more interesting question, is to address the content of the articles supporting most decisions. Focusing on the ten most cited articles of the Statute, it turns out that three main domains are emerging. Firstly, it concerns the fields in which the ICC is innovating, such as the participation of victims (art. 68, cited 250 times) and the rights of the accused (art. 67, cited 201 times, its paragraph 2 being cited 195 times more). A second group of articles refer to the powers of the ICC, the functions and powers of the trial chamber (art. 64, cited 175 


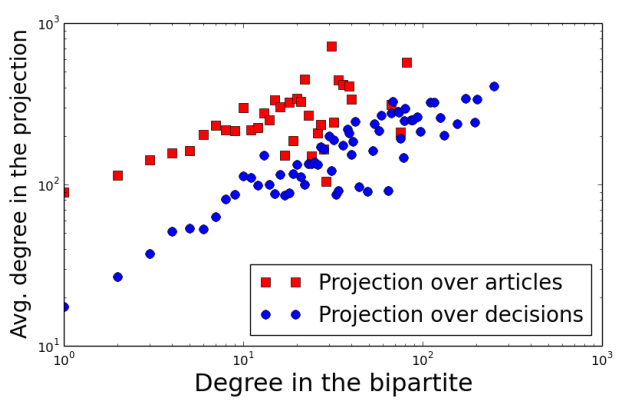

(a) Bipartite vs. Projections

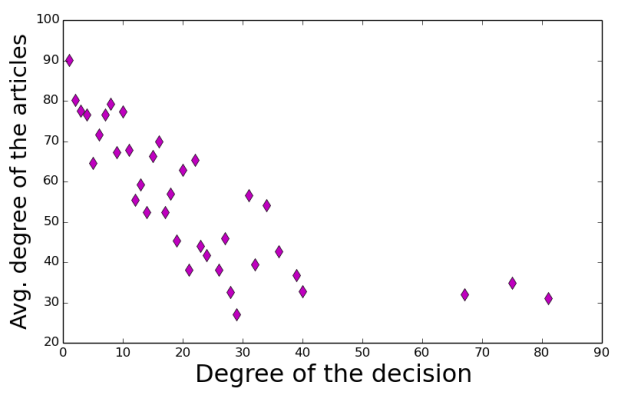

(b) Decisions vs. Articles

Figure 6: Correlation between degrees in the bipartite graph and its projections.

times), the duties and powers of the prosecutor (art. 54 cited 128 times, its paragraph 3-e being cited 156 times more). Finally, the other top cited articles are related to procedural issues, the appeal (art. 82, cited 132 times, its paragraph 1-d being cited 87 times more), the confirmation of charges (art. 61 cited 116 times) and the warrant of arrest (art. 58 cited 110 times).

Overlapping between articles. As discussed in Section 2.2, there is no consensus in the scientific community on the most relevant notion of clustering when analysing bipartite graphs. Two coefficients emerged, namely the bipartite clustering coefficient (Definition 2) and the redundancy coefficient (Definition 4), in the attempt to capture this notion. The first one intends to adapt the notion of clustering coefficient defined for simple graphs by looking at a variation of the triangle motif for bipartite graphs, while the latter intends to see how two $T$ nodes are related to the same set of $\perp$ nodes.

Figure 5(b) shows the inverse cumulative distribution of the two quantities on the bipartite structure of the ICC network. One can see immediately that the two notions behave very differently for this network. While the proportion of nodes with a small clustering coefficient is very large (more than $90 \%$ of the articles have a clustering coefficient lower than 0.3 ), it is the converse for the redundancy. In particular, $35 \%$ of the articles have a redundancy close to 1 . For those articles, it means that if they were not cited by the decisions, the projection of the bipartite structure would remain unchanged. This is a strong property highlighting the fact that when two decisions cite a common article they actually cite the same set of articles, a property which is not captured by the clustering coefficient.

Degree correlations. In order to emphasise the last remark, Figure 6(a) presents some non trivial relations between the degree of the nodes in the bipartite graph and their related degree in the projections (both over articles and over decisions). The two plots show a similar tendency: the more the degree of a node in the bipartite graph, the more its degree (in average) in the projection. As such, this behaviour is not surprising, especially as the progression seems linear ${ }^{15}$. However, looking at the order of magnitude of the axes, the reader might notice that the range of value for the horizontal and vertical axes are very different and thus, pointing out again some overlapping in the bipartite graph. This is particularly obvious for the projection over the decisions. While decisions citing only one article are in average connected to almost 90 other decisions in the projection, this value rises only up to 540 (that is only six times higher) when decisions cite 80 articles. This is clearly due to the fact, as stated earlier, that related decisions tend to rely on the same set of articles.

Figure $6(\mathrm{~b})$ shows the relation between the degree of the decisions in the bipartite graph and the average degree of the related articles. More precisely, a $(x, y)$ dot indicates that if a decision cites $x$ articles, the average degree of those $x$ articles is $y$. The relation between

\footnotetext{
${ }^{15}$ Note that the scale of the axes are logarithmic.
} 


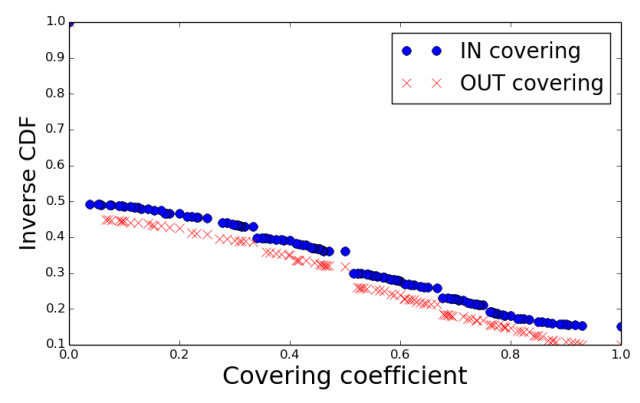

(a) Covering coefficient

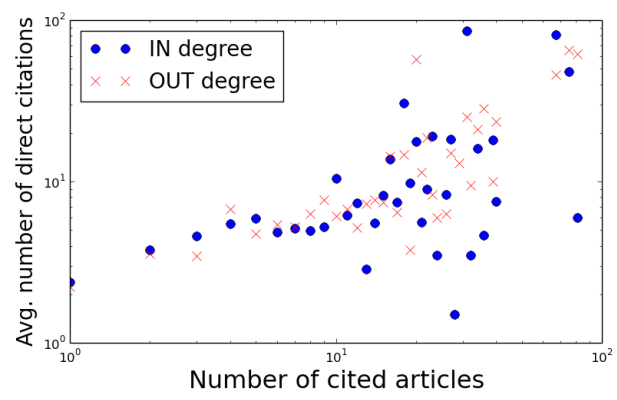

(b) Bipartite vs. direct links

Figure 7: Properties of the hybrid bipartite structure.

those two quantities is surprising. The plot shows that the more the degree of the citation, the lower the average degree of the related articles. Somehow, when a decision cites only few articles, those articles tend to deal with issues that are shared by a lot of other decisions. The case of the 1-degree decision is particularly meaningful. It shows that if a decision relies on a unique article, this article tends to be cited by approximately 90 decisions. On the contrary, if a decision relies on many articles, those tend to be more specific and then less invoked by other decisions.

\subsection{Interplay between the links}

We turn now to the analysis of the hybrid bipartite structure, which enables us to focus on the interplay between the citation of decisions and of articles. We start by the study of the covering coefficient, before investigating more in depth the relation between citing decisions and citing articles in the network.

Covering coefficient. The covering coefficient intends to capture by how much two $\perp$ nodes of hybrid bipartite graph related by a direct citation are also related by a common article they refer to. In other words, this metrics focuses on the fraction of direct links covered by indirect links.

Figure 7(a) shows the inverse cumulative distribution of the in- and -out covering coefficient of the decisions. First, one can see that most of the nodes have their coefficient equal to 0 . Indeed, it is remarkable that only $38.25 \%$ of the direct links are covered by articles. This is a very strong characteristic of the ICC network, which shows that the decisions tend to be procedural and involve chains of cited decisions that deal with technical issues. Yet, investigations showed that this proportion rises to $68.85 \%$ when focusing on decisions which cite at least one article. This shows that, as soon as the decision requires to rely on the articles of the Statute of the Court, those articles tend to indeed cover the direct citations. This observation is strengthened by the fact that, in the opposite case, more than $15 \%$ of the decisions have a coefficient equal to 1 , meaning that all their direct citations are covered by articles.

Relation between citations. In order to better understand the interplay between the two kinds of citations, we present in Figure 7(b) the correlation between the number of articles cited by a decision and the average in- or -out degree of the decision. More precisely, a $(x, y)$ dot means that a decision citing $x$ articles, cites (or is cited by) $y$ decisions in average. While no particular conclusion can be drawn for the in-degrees, the plot highlights a tendency for the out-degrees: the more the number of cited articles, the more the number of cited decisions in average. Going back to the observations made in Section 4.1, this strengthens the conclusion we drew: decisions citing many articles tend to be very specific. As such, the decisions they 


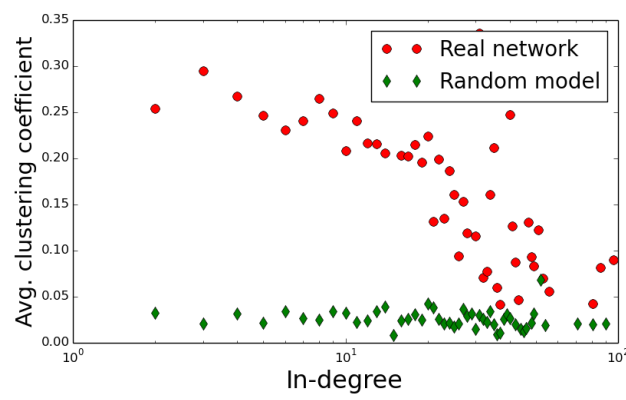

(a) In-degree vs. In-clustering

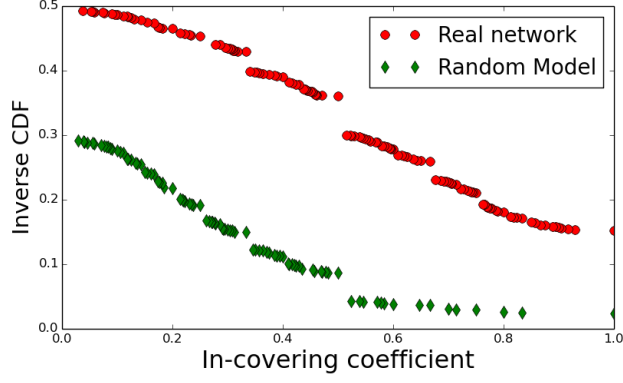

(b) Covering coefficient

Figure 8: Impact of the random model on the structural properties of the network.

directly rely on are likely to focus on the same juridical issues and thus, mechanically leading to a high covering coefficient.

\section{Comparison to a random model}

The analysis conducted in the two previous sections revealed some strong tendencies in the network's structure in the first case of the ICC. While we provided explanation that explains how such findings are related to real juridical issues, it is not yet clear which part of the detected correlations is due to the specific nature this legal network, and which part is induced by the network's point of view adopted in the study.

In order to deepen this question, in this section we compare the previous results to the ones obtained on a structure generated by a simple random model. To that purpose, we apply a natural extension of the standard Configuration Model [3, 8, 28], which basically shuffles all the edges, both in the directed graph and in the bipartite graph. More precisely, the model generates a hybrid bipartite graph with a similar size (same number of nodes and edges) and similar degree distributions. It means that the only structure kept intact by the model is the number of citations a decision has and the number of times an article is cited. By contrast, the end-points of those links are chosen arbitrary. Thus, by analysing the structure of the generated hybrid bipartite graph as we did in Sections 3 and 4, we can investigate how such a random shuffling of the structure impacts the obtained results.

Figure 8 compares the properties of the real and random networks. Figure 8 (a) shows in particular the relation between the in-degree of a citation and its in-clustering coefficient. It is then similar to the blue plot of Figure 4. Regarding those coefficients, the impact of the model is clear. The shuffling has completely deleted the correlation between the two coefficients. Whatever the in-degree of a decision is, its clustering is always around 0.025 , which is in contrast with the conclusions drawn in Section 3.3. This shows that the strong relation observed previously is not trivial and it sustains the juridical perspective we provided to explain the observed correlation.

Although less obvious, Figure 8(b) leads to a similar conclusion. The figure focuses on the covering coefficient and shows the inverse cumulative distribution of this property. It is then similar to the blue plot of Figure $7(\mathrm{a})$. The plot reveals a similar tendency on the model than the one previously observed on the real network. In particular, most of the nodes have their coefficient equal to 0 . However, one can observe that the gap between the two distributions is important. In the extreme case, while more than $15 \%$ of the decisions have a covering coefficient equal to 1 in the real network, only $2 \%$ have such a strong characteristics in the random network. This shows again that such a property is obviously not trivial and it strengthens our conclusions drawn throughout the paper regarding the processes used by the judges to ground their decisions. 


\section{Conclusion and perspectives}

The multiplication of international criminal jurisdictions led the lawyers to recently question the evolution of the interpretation by the judges of international criminal law and of trial proceedings in the international context. Those jurisdictions started providing a rich case-law of juridical decisions which offer the opportunity to use graph theory to analyse and model such datasets. In this paper, we focused on the legal network composed of the decisions taken by the International Criminal Court (ICC), since its creation in 2002. In order to delimit the object under investigation, we focused more particularly on the Lubanga case, which has reached a final verdict.

Legal networks are characterised by the fact that different types of relations relate the decisions. Since judges motivate their decisions by relying either on former decisions, or on articles of the ICC Statute, this induces a two-level network with a rich and intricate notion of relations between the juridical decisions. We proposed in this paper to analyse this network by means of hybrid bipartite graphs that allow to represent the whole information contained in this network. We then assessed the relevance of this approach by analysing different properties of the structure and we validated the outcomes with juridical interpretations of the results.

As a side effect, this approach highlighted the procedural aspect of this jurisdiction and emphasised the key thematic pointed out by the major decisions. Thus, this work converges for the first time two very different approaches to account for the multi-level complexity in legal networks, and presents a unique and appealing opportunity to relate juridical and network sciences.

This work opens the way to many future directions. From a modelling point of view for instance, it leads naturally to the study of a hybrid bipartite model, that would allow to understand which part of the properties highlighted by the present work stem from this specific dataset, and which part would have emerged within a randomised version of the same two-level structure. Several variations can be envisioned with an increasing constraint on the degree correlations. One could, for instance, force the correlation between direct and indirect links to remain stable in the randomising process, or on the contrary, release this constraint. This would provide a rich information on the emergence of the observed properties in hybrid bipartite graphs. This line of research would benefit from previous studies on general models proposed for multi-level networks [34, 42].

Finally, on a broader perspective, one key question that the community needs to address in the future lies in the gathering of data exhibiting similar complex structures. It is indeed often difficult to have at the same time information on bipartite and direct links. For instance, if it is trivial to know which actor plays in which movie (bipartite links), on the contrary it is very difficult to know the exact social ties between the actors (direct links). However, several data involving social networks allowed recently to infer this complex structure. We can cite for instance the Flickr network, where users are related to the photos they comment on (bipartite links), but also to their personal contacts (direct links); or WIKIPEDIA webpages, where articles relate to other articles (direct links), but also label their entry with categories (bipartite links). In the same way, most of legal networks put emphasis on juridical decisions that cite each other, but also rely on law articles, which gather several decisions. All in all, we can expect such intricate structures to be more and more available for formal analyses, such as the one presented in this paper.

\section{Acknowledgements}

This work is partly funded by the National Center for Scientific Research (CNRS) through the PEPS Project "DoRé". The authors are also grateful to Louisa Harutyunyan for her feedback on the paper. 


\section{References}

[1] Tommaso Agnoloni and Ugo Pagallo. The Case Law of the Italian Constitutional Court between Network Theory and Philosophy of Information. In Proceedings of the Second International Workshop on Network Analysis in Law (NAIL 2014), pages 26-38, 2014.

[2] Yong-Yeol Ahn, Sebastian E. Ahnert, James P. Bagrow, and Albert-László Barabási. Flavor network and the principles of food pairing. Nature Scientific Reports, 1:196+, December 2011.

[3] William Aiello, Fan Chung, and Linyuan Lu. A random graph model for massive graphs. In Proceedings of the Thirty-second Annual ACM Symposium on Theory of Computing, STOC '00, pages 171-180, New York, NY, USA, 2000. ACM.

[4] Tim Althoff, Damian Borth, Jörn Hees, and Andreas Dengel. Analysis and Forecasting of Trending Topics in Online Media Streams. In Proceedings of the 21st ACM International Conference on Multimedia, MM '13, pages 907-916, New York, NY, USA, 2013. ACM.

[5] Stefano Battiston and Michele Catanzaro. Statistical properties of corporate board and director networks. European Physics Journal B, 38(2):345-352, 2004.

[6] Elisa Bellotti. Getting funded. Multi-level network of physicists in Italy. Social Networks, $34(2): 215-229,2012$.

[7] Emilia Bellucci, Arno R. Lodder, and John Zeleznikow. Integrating artificial intelligence, argumentation and game theory to develop an online dispute resolution environment. In 16th IEEE International Conference on Tools with Artificial Intelligence (ICTAI), pages 749-754. IEEE Computer Society, 2004.

[8] Béla Bollobás. Random graphs, volume 73 of Cambridge Studies in Advanced Mathematics. Cambridge University Press, Cambridge, second edition, 2001.

[9] Romain Boulet, Pierre Mazzega, and Danièle Bourcier. A network approach to the french system of legal codes - part i: analysis of a dense network. Artificial Intelligence and Law, 19(4):333-355, 2011.

[10] Danièle Bourcier and Pierre Mazzega. Codification, law article and graphs. In Arno R. Lodder and Laurens Mommers, editors, Legal Knowledge and Information Systems (JURIX), volume 165 of Frontiers in Artificial Intelligence and Applications, pages 29-38. IOS Press, 2007.

[11] Antonio Cassese, Guido Acquaviva, and Alex Whiting. International Criminal Law: Cases and Commentary. Oxford University Press, 2011.

[12] Seth J Chandler. The Network Structure of Supreme Court Jurisprudence. The Mathematica Journal, 10(3):501-526, 2005.

[13] James R. Clough, Jamie Gollings, Tamar V. Loach, and Tim S. Evans. Transitive reduction of citation networks. Journal of Complex Networks, 3(2):189-203, 2015.

[14] M G Everett and S P Borgatti. The dual-projection approach for two-mode networks. Social Networks, 35(2):204-210, 2013.

[15] Ramon Ferrer and Ricard V. Solé. The small world of human language. Proceedings of The Royal Society of London., 268:2261-2266, 2001.

[16] Fabrice Le Fessant, Sidath B. Handurukande, Anne-Marie Kermarrec, and Laurent Massoulié. Clustering in peer-to-peer file sharing workloads. In Geoffrey M. Voelker and Scott Shenker, editors, 3-rd International workshop on Peer-To-Peer Systems (IPTPS), volume 3279 of Lecture Notes in Computer Science, pages 217-226. Springer, 2004. 
[17] James H Fowler and Sangick Jeon. The authority of Supreme Court precedent. Social Networks, 30(1):16-30, 2008.

[18] Gilbert Guillaume. The use of precedents by International Judges and Arbitrators. Journal of International Dispute Settlement, 2(1):5-23, 2011.

[19] Jean-Loup Guillaume and Matthieu Latapy. Bipartite graphs as models of complex networks. Physica A: Statistical and Theoretical Physics, 371(2):795-813, November 2006.

[20] Marios Koniaris, Ioannis Anagnostopoulos, and Yannis Vassiliou. Network Analysis in the Legal Domain: A complex model for European Union legal sources. CoRR, abs/1501.0, 2015 .

[21] Robert Kowalski and Marek Sergot. The Use of Logical Models in Legal Problem Solving*. Ratio Juris, 3(2):201-218, 1990.

[22] David Krackhardt and MarkS. Handcock. Heider vs simmel: Emergent features in dynamic structures. In Edoardo Airoldi, DavidM. Blei, StephenE. Fienberg, Anna Goldenberg, EricP. Xing, and AliceX. Zheng, editors, Statistical Network Analysis: Models, Issues, and New Directions, volume 4503 of Lecture Notes in Computer Science, pages 14-27. Springer Berlin Heidelberg, 2007.

[23] Matthieu Latapy, Clémence Magnien, and Nathalie Del Vecchio. Basic notions for the analysis of large two-mode networks. Social Networks, 30(1):31 - 48, 2008.

[24] Emmanuel Lazega, Marie-Thérèse Jourda, Lise Mounier, and Rafaël Stofer. Catching up with big fish in the big pond? Multi-level network analysis through linked design. Social Networks, 30(2):159-176, 2008.

[25] Arno R Lodder and Aimée Herczog. DiaLaw: A Dialogical Framework for Modeling Legal Reasoning. In Proceedings of the 5th International Conference on Artificial Intelligence and Law, ICAIL '95, pages 146-155, New York, NY, USA, 1995. ACM.

[26] Shmoolik Mangan and Uri Alon. Structure and function of the feed-forward loop network motif. PNAS, 100(21):11980-11985, October 2003.

[27] Thom Neale. Citation Analysis of Canadian Case Law. Journal of Open Access to Law, $1(1): 1-51,2013$.

[28] Mark Newman, Steven H. Strogatz, and Duncan J. Watts. Random graphs with arbitrary degree distributions and their applications. Physical Review E, 64(2):026118, 2001.

[29] Mark Newman, Duncan J. Watts, and Steven H. Strogatz. Random graph models of social networks. PNAS, 99:2566-2572, 2002.

[30] Tore Opsahl. Triadic closure in two-mode networks: Redefining the global and local clustering coefficients. Social Networks, 35(2):159-167, 2013.

[31] Thomas Schank and Dorothea Wagner. Approximating clustering coefficient and transitivity. Journal of Graph Algorithms and Applications, 9(2):265-275, 2005.

[32] Thomas Schank and Dorothea Wagner. Finding, counting and listing all triangles in large graphs, an experimental study. In Sotiris E. Nikoletseas, editor, Workshop on Experimental and Efficient Algorithms (WEA), volume 3503 of Lecture Notes in Computer Science, pages 606-609. Springer, 2005.

[33] Thomas A Smith. The Web of Law. San Diego L. Rev., 44:309, 2007.

[34] Tom A B Snijders, Alessandro Lomi, and Vanina Jasmine Torló. A model for the multiplex dynamics of two-mode and one-mode networks, with an application to employment preference, friendship, and advice. Social Networks, 35(2):265-276, 2013. 
[35] Andrew Stranieri and John Zeleznikow. Knowledge Discovery from Legal Databases. Springer, 1st edition, 2005.

[36] Fabien Tarissan. Comparing Overlapping Properties of Real Bipartite Networks. In Ali Sanayei, Otto E. Rössler, and Ivan Zelinka, editors, ISCS 2014: Interdisciplinary Symposium on Complex Systems, volume 14 of Emergence, Complexity and Computation, pages 309-317. Springer, 2015.

[37] Fabien Tarissan and Raphälle Nollez-Goldbach. The network of the International Criminal Court decisions as a complex system. In A. Sanayei, I. Zelinka, and O. E. Rossler, editors, ISCS 2013: Interdisciplinary Symposium on Complex Systems, volume 8 of Emergence, Complexity and Computation, pages 255-264. Springer, 2013.

[38] Fabien Tarissan, Bruno Quoitin, Pascal Mérindol, Benoit Donnet, Jean-Jacques Pansiot, and Matthieu Latapy. Towards a bipartite graph modeling of the internet topology. Computer Networks, 57(11):2331-2347, 2013.

[39] Vincent A Traag, Ridho Reinanda, and Gerry van Klinken. Structure of an elite cooccurrence network. arXiv preprint arXiv:1409.1744, 2014.

[40] Michele Tumminello, Salvatore Miccichè, Fabrizio Lillo, Jyrki Piilo, and Rosario N. Mantegna. Statistically validated networks in bipartite complex systems. PLoS One, 6(3):e17994, August 2011.

[41] A Vázquez, R Dobrin, D Sergi, J.-P. Eckmann, Z N Oltvai, and Albert-László Barabási. The topological relationship between the large-scale attributes and local interaction patterns of complex networks. Proceedings of the National Academy of Sciences, 101(52):17940-17945, 2004.

[42] Peng Wang, Philippa Pattison, and Garry Robins. Exponential random graph model specifications for bipartite networks - A dependence hierarchy. Social Networks, 35(2):211$222,2013$.

[43] Stanley Wasserman and Dawn Iacobucci. Statistical modelling of one-mode and twomode networks: Simultaneous analysis of graphs and bipartite graphs. British Journal of Mathematical and Statistical Psychology, 44(1):13-43, 1991.

[44] Duncan J. Watts and Steven H. Strogatz. Collective dynamics of small-world networks. Nature, 393(6684):440-442, june 1998.

[45] Ryan Whalen. Modeling Annual Supreme Court Influence: The Role of Citation Practices and Judicial Tenure in Determining Precedent Network Growth. In Ronaldo Menezes, Alexandre Evsukoff, and Marta C González, editors, Complex Networks, volume 424 of Studies in Computational Intelligence, pages 169-176. Springer Berlin Heidelberg, 2013.

[46] Richard J Williams, Eric L Berlow, Jennifer A Dunne, Albert-László Barabási, and Neo D Martinez. Two degrees of separation in complex food webs. Proceedings of the National Academy of Sciences, 99(20):12913-12916, 2002.

[47] Radboud Winkels, Jelle de Ruyter, and Henryk Kroese. Determining Authority of Dutch Case Law. In Katie Atkinson, editor, Legal Knowledge and Information Systems (JURIX), volume 235 of Frontiers in Artificial Intelligence and Applications, pages 103-112. IOS Press, 2011.

[48] Jaewon Yang and Jure Leskovec. Defining and evaluating network communities based on ground-truth. Knowledge and Information Systems, 42(1):181-213, 2015.

[49] John Zeleznikow. Building decision support systems in discretionary legal domains. International Review of Law, Computers \& Technology, 14(3):341-356, 2000.

[50] John Zeleznikow and Daniel Hunter. Building Intelligent Legal Information Systems: Representation and Reasoning in Law, volume 13 of Computer / Law. Kluwer Law and Taxation Publishers, 1994. 